\title{
$G$-constellations and the maximal resolution of a quotient surface singularity
}

\author{
Akira IsHII \\ (Received February 18, 2020) \\ (Revised June 30, 2020)
}

\begin{abstract}
For a finite subgroup $G$ of $\mathrm{GL}(2, \mathbb{C})$, we consider the moduli space $\mathscr{M}_{\theta}$ of $G$-constellations. It depends on the stability parameter $\theta$ and if $\theta$ is generic it is a resolution of singularities of $\mathbb{C}^{2} / G$. In this paper, we show that a resolution $Y$ of $\mathbb{C}^{2} / G$ is isomorphic to $\mathscr{M}_{\theta}$ for some generic $\theta$ if and only if $Y$ is dominated by the maximal resolution under the assumption that $G$ is abelian or small.
\end{abstract}

\section{Introduction}

The moduli spaces of $G$-constellations (on an affine space) are introduced in [CI04]. It is a generalization of the Hilbert scheme of $G$-orbits, which is denoted by $G$-Hilb. The moduli space depends on some stability parameter $\theta$ and the moduli space of $\theta$-stable $G$-constellations is denoted by $\mathscr{M}_{\theta}$. If $G$ is a subgroup of $\operatorname{SL}(n, \mathbb{C})$ acting on $\mathbb{C}^{n}$ and $n \leq 3$, then $\mathscr{M}_{\theta}$ is a crepant resolution of $\mathbb{C}^{n} / G$ for a generic stability parameter $\theta$. The main result of [CI04] is that for a finite abelian subgroup $G \subset \operatorname{SL}(3, \mathbb{C})$ and for a projective crepant resolution $Y \rightarrow \mathbb{C}^{3} / G$, there is a generic stability parameter $\theta$ such that $Y \cong \mathscr{M}_{\theta}$. See [Kę14], [NdCS17], [Jun16] and [Jun18] for related results.

The purpose of this paper is to consider the case where $G$ is a finite subgroup of $\operatorname{GL}(2, \mathbb{C})$. In this case, $G$-Hilb $\left(\mathbb{C}^{2}\right)$ is the minimal resolution of $\mathbb{C}^{2} / G$ by [Ish02] but $\mathscr{M}_{\theta}$ is a resolution which may not be minimal for generic $\theta$ (as we see in this paper). Then what is the condition for a resolution $Y \rightarrow \mathbb{C}^{2}$ to be isomorphic to some $\mathscr{M}_{\theta}$ ? One important observation is that there is a fully faithful functor (see Theorem 3)

$$
D^{b}\left(\operatorname{coh} \mathscr{M}_{\theta}\right) \hookrightarrow D^{b}\left(\operatorname{coh}^{G} \mathbb{C}^{2}\right)
$$

between the derived categories. According to the DK hypothesis [Kaw18], the inclusion of derived categories should be related with inequalities of canonical divisors. Then it is natural to ask if the following is true: $Y$ is isomorphic to $\mathscr{M}_{\theta}$ for some $\theta$ if and only if $Y$ is between the minimal and the maximal

2010 Mathematics Subject Classification. 14D20; 14E16; $14 \mathrm{~J} 17$.

Key words and phrases. G-constellation, quotient singularity, maximal resolution. 
resolutions (see Conjecture 4), where the maximal resolution means the unique maximal one satisfying the inequality as in [KSB88]. The main result of this paper is the following. Recall that $G$ is said to be small if it contains no pseudo reflection.

TheOREM 1 (= Theorem 7). Let $G \subset \mathrm{GL}(2, \mathbb{C})$ be a finite small subgroup and let $X=\mathbb{C}^{2} / G$ be the quotient singularity. Then a resolution of singularities $Y \rightarrow X$ is isomorphic to $\mathscr{M}_{\theta}$ for some $\theta$ if and only if $Y$ is dominated by the maximal resolution.

Conjecture 4 is a conjecture for general (not necessarily small) finite subgroups where the maximal resolution is defined for the pair of the quotient variety $\mathbb{C}^{2} / G$ and the associated boundary divisor. The "only if" part of the conjecture is proved in Proposition 1 by using the embedding of $G$ into $\operatorname{SL}(3, \mathbb{C})$ and the fact that the moduli space of $G$-constellations for $G \subset$ $\operatorname{SL}(3, \mathbb{C})$ is a crepant resolution of $\mathbb{C}^{3} / G$. We can show that the conjecture is true if $G$ is abelian (Theorem 5) by using the result of [CI04]. The idea in the non-abelian case of Theorem 1 is to use iterated construction of moduli spaces as in [IINdC13] and reduce the problem to the abelian group case. Namely, let $N$ be the cyclic group generated by $-I$, which is a normal subgroup of every non-abelian finite small subgroup. We consider $G / N$-constellations on the moduli space of $N$-constellations in $\S 7$. In order to do such iterated constructions, we define $G$-constellations on a general variety and consider their stability parameters in $\S 6$. A key to the proof of Theorem 1 is the description of the space of stability parameters for $G / N$-constellations on the moduli space of $N$-constellations, which is done in $\S 8.1$. The proof of Theorem 1 is completed in $\S 8.2$.

\section{Acknowledgements}

This work depends a lot on the joint work [CI04] and the author thanks Alastair Craw for stimulating discussions since then. He is grateful to SeungJo Jung and the anonymous referee for many useful comments. This research was supported in part by JSPS KAKENHI Grant Number 15K04819.

\section{2. $G$-constellations on $\mathbb{C}^{n}$}

2.1. Definitions. Let $V=\mathbb{C}^{n}$ be an affine space and $G \subset \mathrm{GL}(V)$ a finite subgroup.

Definition 1. A $G$-constellation on $V$ is a $G$-equivariant coherent sheaf $E$ on $V$ such that $H^{0}(E)$ is isomorphic to the regular representation of $G$ as a $\mathbb{C}[G]$-module. 
Let $R(G)=\bigoplus_{\rho \in \operatorname{Irr}(G)} \mathbb{Z} \rho$ be the representation ring of $G$, where $\operatorname{Irr}(G)$ denotes the set of irreducible representations of $G$. The parameter space of stability conditions of $G$-constellations is the $\mathbb{Q}$-vector space

$$
\Theta=\left\{\theta \in \operatorname{Hom}_{\mathbb{Z}}(R(G), \mathbb{Q}) \mid \theta(\mathbb{C}[G])=0\right\},
$$

where $\mathbb{C}[G]$ is regarded as the regular representation of $G$. The definition of the stability is based on the stability of quiver representations [Kin94]:

Definition 2. A $G$-constellation $E$ is $\theta$-stable (or $\theta$-semistable) if every proper G-equivariant coherent subsheaf $0 \subsetneq F \subsetneq E$ satisfies $\theta\left(H^{0}(F)\right)>0$ (or $\left.\theta\left(H^{0}(F)\right) \geq 0\right)$. Here the representation space $H^{0}(F)$ of $G$ is regarded as an element of $R(G)$.

By virtue of King [Kin94], there is a fine moduli scheme $\mathscr{M}_{\theta}=\mathscr{M}_{\theta}(V)$ of $\theta$-stable $G$-constellations on $V$.

Definition 3. We say that a parameter $\theta \in \Theta$ is generic if a $\theta$-semistable $G$-constellation is always $\theta$-stable.

There is a morphism $\tau: \mathscr{M}_{\theta}(V) \rightarrow V / G$ which sends a $G$-constellation to its support. It is a projective morphism if $\theta$ is generic (see [CI04, Proposition $2.2])$.

2.2. Results of [CI04]. In this subsection, we recall results from [CI04]. Suppose $V=\mathbb{C}^{3}$ and let $G \subset \mathrm{SL}(V)$ be a finite abelian subgroup. For a generic parameter $\theta \in \Theta$, the morphism

$$
\tau: \mathscr{M}_{\theta} \rightarrow \mathbb{C}^{3} / G
$$

is a projective crepant resolution and we have a Fourier-Mukai transform

$$
\Phi_{\theta}: D^{b}\left(\operatorname{coh} \mathscr{M}_{\theta}\right) \stackrel{\sim}{\rightarrow} D^{b}\left(\operatorname{coh}^{G}\left(\mathbb{C}^{3}\right)\right) .
$$

Here for a variety $Y$, coh $Y$ denotes the category of coherent sheaves on $Y$ and if $Y$ is acted on by a finite group $G, \operatorname{coh}^{G}(Y)$ denotes the category of $G$-equivariant coherent sheaves on $Y$. The subset of $\Theta$ consisting of generic parameters is divided into chambers; the moduli space $\mathscr{M}_{\theta}$ and the equivalence $\Phi_{\theta}$ depend only on the chamber to which $\theta$ belongs. Thus we write $\mathscr{M}_{C}$ and $\Phi_{C}$ instead of $\mathscr{M}_{\theta}$ and $\Phi_{\theta}$ where $C$ is the chamber that contains $\theta$. We write

$$
\varphi_{C}: K\left(\operatorname{coh}_{0} \mathscr{M}_{C}\right) \rightarrow K\left(\operatorname{coh}_{0}^{G}\left(\mathbb{C}^{3}\right)\right)
$$

for the induced isomorphism of the Grothendieck groups of the full subcategories $\operatorname{coh}_{0} \mathscr{M}_{\theta}$ and $\operatorname{coh}_{0}^{G}\left(\mathbb{C}^{3}\right)$ consisting of sheaves supported on the sub- 
sets $\tau^{-1}(0)$ and on $\{0\}$ respectively. Since $K\left(\operatorname{coh}_{0}^{G}\left(\mathbb{C}^{3}\right)\right)$ has a basis consisting of skyscraper sheaves $\mathcal{O}_{0} \otimes \rho$ with $\rho \in \operatorname{Irr}(G)$, it is naturally identified with $R(G)$.

The dual of $\varphi_{C}$ is regarded as the map

$$
\varphi_{C}^{*}: K\left(\operatorname{coh}^{G}\left(\mathbb{C}^{3}\right)\right) \rightarrow K\left(\operatorname{coh} \mathscr{M}_{\theta}\right)
$$

between the Grothendieck groups of the categories of sheaves without restrictions on the supports. Then $K\left(\operatorname{coh}^{G}\left(\mathbb{C}^{3}\right)\right)$ is identified with $\operatorname{Hom}(R(G), \mathbb{Z})$ and $\varphi_{C}^{*}$ induces an isomorphism

$$
\Theta \stackrel{\sim}{\rightarrow} F^{1} K\left(\operatorname{coh} \mathscr{M}_{\theta}\right)_{\mathbb{Q}}
$$

where $F^{i} K\left(\operatorname{coh} \mathscr{M}_{\theta}\right)$ is the subgroup consisting of the classes of objects whose supports are at least of codimension $i$.

On $\mathscr{M}_{C}$ there are tautological bundles $\mathscr{R}_{\rho}$ for irreducible representations $\rho$ such that $\bigoplus_{\rho} \mathscr{R}_{\rho} \otimes_{\mathbb{C}} \rho$ has a structure of the universal $G$-constellation. For $\theta \in C$,

$$
\mathscr{L}_{C}(\theta):=\bigotimes_{\rho}\left(\operatorname{det} \mathscr{R}_{\rho}\right)^{\otimes \theta(\rho)}
$$

is the (fractional) ample line bundle on $\mathscr{M}_{\theta}$ obtained by the GIT construction. It coincides with the class

$$
\left[\varphi_{C}^{*}(\theta)\right] \in F^{1} K\left(\operatorname{coh} \mathscr{M}_{C}\right)_{\mathbb{Q}} / F^{2} K\left(\operatorname{coh} \mathscr{M}_{C}\right)_{\mathbb{Q}} \cong \operatorname{Pic}\left(\mathscr{M}_{C}\right)_{\mathbb{Q}}
$$

as in $[\mathrm{CI04}, \S 5.1]$. Hence $\left[\varphi_{C}^{*}(\theta)\right] \in \operatorname{Amp}\left(\mathscr{M}_{C}\right)$ where $\operatorname{Amp}\left(\mathscr{M}_{C}\right)$ is the ample cone considered in $\operatorname{Pic}\left(\mathscr{M}_{C}\right)_{\mathbb{Q}}$. The main theorem of [CI04] and the argument in $[\mathrm{CI} 04, \S 8]$ show the following:

THEOREM 2 ([CI04]). For any projective crepant resolution $Y \rightarrow \mathbb{C}^{3} / G$ and a class $l \in \operatorname{Amp}(Y)$, there exist a chamber $C$ with $Y \cong \mathscr{M}_{C}$ and a parameter $\theta \in C$ satisfying $l=\left[\varphi_{C}^{*}(\theta)\right]$.

Proof. The existence of a chamber $C$ such that $Y \cong \mathscr{M}_{C}$ is [CI04, Theorem 1.1]. Moreover, [CI04, Proposition 8.2] ensures that we can find a chamber $C$ and a parameter $\theta \in \bar{C}$ with $l=\left[\varphi_{C}^{*}(\theta)\right]$. Suppose $\theta \in \bar{C} \backslash C$. We have to see we can perturb $\theta$ in the fiber of $p \circ \varphi_{C}^{*}$ so that $\theta$ is in some chamber, where

$$
p: F^{1} K\left(\operatorname{coh} \mathscr{M}_{C}\right)_{\mathbb{Q}} \rightarrow \operatorname{Pic}\left(\mathscr{M}_{C}\right)_{\mathbb{Q}}
$$

is the projection. Here recall that a wall of the chamber $C$ is either the preimage of a wall of the ample cone by $p \circ \varphi_{C}^{*}$ (type I or III) or does not contain a fiber of $p \circ \varphi_{C}^{*}$ (type 0 ); see [CI04, Theorem 5.9]. In our case, $p \circ \phi_{C}^{*}(\theta)=l$ 
is ample and therefore $\theta$ is on walls of type 0 . Since the images of adjacent chambers in $F^{1} K\left(\operatorname{coh} \mathscr{M}_{C}\right)_{\mathbb{Q}}$ are related as in [CI04, (8.2) or (8.3)], we can perturb $\theta$ in the fiber of $p \circ \varphi_{C}^{*}$ and go out of walls.

2.3. $G$-constellations on $\mathbb{C}^{2}$. Let $G$ be a finite subgroup of $\operatorname{GL}(2, \mathbb{C})$.

THEOREM 3. If $\theta$ is generic, then the moduli space $\mathscr{M}_{\theta}$ is a resolution of singularities of $\mathbb{C}^{2} / G$. Moreover, the universal family of $G$-constellations defines a fully faithful functor

$$
\Phi_{\theta}: D^{b}\left(\operatorname{coh} \mathscr{M}_{\theta}\right) \rightarrow D^{b}\left(\operatorname{coh}^{G} \mathbb{C}^{2}\right) .
$$

Proof. This is essentially Theorem 1.3 in the first arXiv version of [BKR01]. We have the inequality

$$
\operatorname{dim} \mathscr{M}_{\theta} \times_{\left(\mathbb{C}^{2} / G\right)} \mathscr{M}_{\theta} \leq \operatorname{dim} \mathbb{C}^{2}
$$

which is sharper than the assumption in [BKR01]. This allows us to apply the argument of [BKR01] (without using the triviality of the Serre functors) to show that $\Phi_{\theta}$ is fully faithful and that $\mathscr{M}_{\theta}$ is smooth and connected (see [Ish02, Theorem 6.2]).

The problem we consider is to characterize the resolutions $Y$ such that $Y \cong \mathscr{M}_{\theta}$ for some generic $\theta$.

\section{The maximal resolution}

Let $G$ be a finite subgroup of $\operatorname{GL}(2, \mathbb{C})$, which is not necessarily small, i.e., the action may not be free on $\mathbb{C}^{2} \backslash\{0\}$. Then the quotient variety $X=\mathbb{C}^{2} / G$ is equipped with a boundary divisor $B$ determined by the equality $\pi^{*}\left(K_{X}+B\right)=$ $K_{\mathbb{C}^{2}}$. More precisely, $B$ is expressed as

$$
B=\sum_{j} \frac{m_{j}-1}{m_{j}} B_{j},
$$

where $B_{j} \subset X$ is the image of a one-dimensional linear subspace whose pointwise stabilizer subgroup $G_{j} \subset G$ is cyclic of order $m_{j}$. Note that $G$ is small if and only if $B=0$. Let $\tau: Y \rightarrow X$ be a resolution of singularities and write

$$
K_{Y}+\tau_{*}^{-1} B \equiv \tau^{*}\left(K_{X}+B\right)+\sum_{i} a_{i} E_{i}
$$

where $E_{i}$ are the exceptional divisors and $a_{i} \in \mathbb{Q}$. Recall that $(X, B)$ is a $\operatorname{KLT}$ pair ([KM98, Proposition 5.20]), which implies $a_{i}>-1$ for all $i$. Then among 
the resolutions $Y$ which satisfy $a_{i} \leq 0$ for all $i$, there is a unique maximal one, as in [KSB88] (see also [Kaw18, Theorem 17]). It is called the maximal resolution of $(X, B)$ and we denote it by $Y_{\max }$.

Notice that the system of inequalities $a_{i} \leq 0$ is an inequality between canonical divisors. According to the DK-hypothesis [Kaw18], the inequality should correspond to the embedding of derived categories in Theorem 3 with $Y=\mathscr{M}_{\theta}$. Thus we make the following conjecture:

CONJECTURE 4. Let $G \subset \mathrm{GL}(2, \mathbb{C})$ be a finite subgroup and consider the quotient $X=\mathbb{C}^{2} / G$ with the boundary divisor $B$. For any resolution of singularities $Y \rightarrow X$, there is a generic $\theta \in \Theta$ with $Y \cong \mathscr{M}_{\theta}$ if and only if there is a morphism $Y_{\max } \rightarrow Y$ over $X$. Here $Y_{\max }$ is the maximal resolution of $(X, B)$.

\section{4. "Only if" part}

In this section, we show the "only if" part of Conjecture 4. Embed $\mathrm{GL}(2, \mathbb{C})$ into $\mathrm{SL}(3, \mathbb{C})$ by sending a matrix $A \in \mathrm{GL}(2, \mathbb{C})$ to $\left(\begin{array}{cc}A & 0 \\ 0 & \operatorname{det}(A)^{-1}\end{array}\right)$. Then for $\theta \in \Theta$, we can consider the moduli space $\mathscr{M}_{\theta}\left(\mathbb{C}^{3}\right)$ of $\theta$-stable $G$-constellations on $\mathbb{C}^{3}$ with respect to the action of $G$ on $\mathbb{C}^{3}$.

Lemma 1. For any $\theta \in \Theta$, there is a closed embedding $\mathscr{M}_{\theta} \hookrightarrow \mathscr{M}_{\theta}\left(\mathbb{C}^{3}\right)$ which fits into the commutative diagram

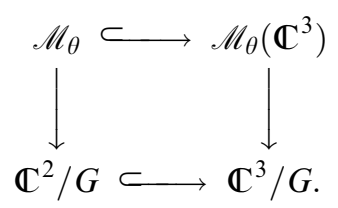

Moreover, if $\theta$ is generic for $G$-constellations on $\mathbb{C}^{3}$, then the vertical arrows are projective and hence are resolutions of singularities.

Proof. Recall that the universal family of $G$-constellations on $\mathbb{C}^{3}$ is given by the tautological bundles $\left\{\mathscr{R}_{\rho}\right\}_{\rho \in \operatorname{Irr} G}$ and the $G$-equivariant morphism

$$
\bigoplus_{\rho} \mathscr{R}_{\rho} \otimes_{\mathbb{C}} \rho \rightarrow \mathbb{C}^{3} \otimes\left(\bigoplus_{\rho} \mathscr{R}_{\rho} \otimes_{\mathbb{C}} \rho\right) .
$$

If $\rho_{\text {nat }}$ denotes the representation given by $G \subset \mathrm{GL}(2, \mathbb{C})$, then $\mathbb{C}^{3}$ above is $\rho_{\text {nat }} \oplus \operatorname{det} \rho_{\text {nat }}^{*}$. Taking the third coordinate of $\mathbb{C}^{3}$ in (4.1) we obtain a morphism

$$
z_{\rho}: \mathscr{R}_{\rho} \rightarrow \mathscr{R}_{\rho \otimes \operatorname{det} \rho_{\text {nat }}}
$$


for each $\rho$. It is straightforward that the scheme theoretic intersection of the zero loci of $z_{\rho}$ 's is isomorphic to $\mathscr{M}_{\theta}$. Hence $\mathscr{M}_{\theta}$ is a closed subscheme of $\mathscr{M}_{\theta}\left(\mathbb{C}^{3}\right)$. Moreover, we can see that the composite $\mathscr{M}_{\theta} \hookrightarrow \mathscr{M}_{\theta}\left(\mathbb{C}^{3}\right) \rightarrow \mathbb{C}^{3} / G$ factors through $\mathbb{C}^{2} / G$. If $\theta$ is generic for $G$-constellations on $\mathbb{C}^{3}$, then it is also generic for $G$-constellations on $\mathbb{C}^{2}$, from which the projectivities of the vertical arrows follow.

Now let us prove the "only if" part.

Proposition 1. If $\theta$ is generic, then there is a morphism $Y_{\max } \rightarrow \mathscr{M}_{\theta}$ over $X$.

Proof. Putting $Y=\mathscr{M}_{\theta}$, we show that $a_{i} \leq 0$ for all $i$ in (3.1). Embed $G$ into $\operatorname{SL}(3, \mathbb{C})$ and consider $U:=\mathscr{M}_{\theta}\left(\mathbb{C}^{3}\right)$, the moduli space of $\theta$-stable $G$-constellations on $\mathbb{C}^{3}$. Here, we may assume that $\theta$ is generic for $G$ constellations on $\mathbb{C}^{3}$ by slightly perturbing $\theta$ if necessary. Then $U$ is a crepant resolution of $\mathbb{C}^{3} / G$ containing $Y$ by Lemma 1 and therefore we have

$$
\left.K_{Y} \cong \mathcal{O}_{U}(Y)\right|_{Y}
$$

Let $z$ be the coordinate function of $\mathbb{C}^{3}$ such that $\mathbb{C}^{2} \subset \mathbb{C}^{3}$ is defined by $z=0$. Then $z^{n}$ is invariant under the action of $G$ where $n$ is the order of $G$. We claim that the principal divisor $\left(z^{n}\right)$ on $U$ is of the form

$$
\left(z^{n}\right)=n Y+\sum_{j} \frac{n\left(m_{j}-1\right)}{m_{j}} B_{j}^{\prime}+\sum_{k} d_{k} D_{k}
$$

where $B_{j}^{\prime}, D_{k} \subset U$ are prime divisors such that $B_{j}^{\prime} \cap Y=\tau_{*}^{-1} B_{j}$ and $D_{k} \cap Y$ is contained in the exceptional locus of $Y \rightarrow \mathbb{C}^{2} / G$ (or empty). This is saying that there exists an exceptional prime divisor $B_{j}^{\prime}$ of $U \rightarrow \mathbb{C}^{3} / G$ lying over $B_{j}$ with $B_{j}^{\prime} \cap Y=\tau_{*}^{-1} B_{j}$ and that its coefficient in $\left(z^{n}\right)$ is $\frac{n\left(m_{j}-1\right)}{m_{j}}$. We may check this over the complete local ring $\hat{\mathcal{O}}_{\mathbb{C}^{3} / G, P}$ at a point $P \in B_{j} \backslash\{0\}$. Since $G_{j}$ is the stabilizer subgroup of a point of $\mathbb{C}^{3}$ lying over $P$, there is an isomorphism of complete local rings:

$$
\hat{\mathcal{O}}_{\mathbb{C}^{3} / G, P} \cong \hat{\mathcal{O}}_{\mathbb{C}^{3} / G_{j},[0]} .
$$

Let $\tilde{B}_{j}$ be a line in $\mathbb{C}^{2}$ mapped to $B_{j}$ and take a $G_{j}$-invariant linear subspace $\tilde{B}_{j}^{\perp}$ of $\mathbb{C}^{3}$ such that

$$
\mathbb{C}^{3}=\tilde{B}_{j} \times \tilde{B}_{j}^{\perp} .
$$

Then $G_{j} \cong \mathbb{Z} / m_{j} \mathbb{Z}$ is a subgroup of $\{1\} \times \operatorname{SL}\left(\tilde{B}_{j}^{\perp}\right)$ and therefore we have

$$
\mathbb{C}^{3} / G_{j} \cong \tilde{B}_{j} \times\left(\tilde{B}_{j}^{\perp} / G_{j}\right)
$$


where $\tilde{B}_{j}^{\perp} / G_{j}$ is a rational double point of type $A_{m_{j}-1}$. Thus we can see that on the crepant resolution

$$
U \times_{\left(\mathbb{C}^{3} / G\right)} \operatorname{Spec} \hat{\mathcal{O}}_{\mathbb{C}^{3} / G, P} \rightarrow \operatorname{Spec} \hat{\mathcal{O}}_{\mathbb{C}^{3} / G, P} \cong \operatorname{Spec} \hat{\mathcal{O}}_{\mathbb{C}^{3} / G_{j},[0]},
$$

there is a prime divisor $\hat{B}_{j}^{\prime}$ with desired properties such that the coefficient of $\hat{B}_{j}^{\prime}$ in the divisor $\left(z^{m_{j}}\right)$ is $m_{j}-1$. Since $m_{j}$ divides $n$, this proves (4.3).

From (4.2) and (4.3), we obtain

$$
K_{Y}+\tau_{*}^{-1} B \equiv-\sum \frac{d_{k}}{n}\left(D_{k} \cap Y\right) .
$$

Here, note that $z^{n}$ is a regular function and therefore the coefficients in (4.3) are all non-negative. Especially, we have $d_{k} \geq 0$ for all $k$. This proves the assertion since $K_{X}+B \in \operatorname{Pic}(X) \otimes \mathbb{Q}=0$ in (3.1).

\section{Abelian group case}

Let $G \subset \operatorname{GL}(2, \mathbb{C})$ be a finite abelian subgroup of order $n$. As in the previous section, we embed $G \subset \mathrm{GL}(2, \mathbb{C})$ into $\operatorname{SL}(3, \mathbb{C})$.

THEOREM 5. Conjecture 4 is true if $G$ is abelian.

Proof. It is sufficient to prove the "if" part by Proposition 1. Let $Y \rightarrow X=\mathbb{C}^{2} / G$ be a resolution which is dominated by $Y_{\max }$. By Proposition 2 below, there is a projective crepant resolution $U \rightarrow \mathbb{C}^{3} / G$ such that $Y \subset U$. Then $[\mathrm{CI04}]$ ensures that there is a generic parameter $\theta$ such that $U \cong \mathscr{M}_{\theta}\left(\mathbb{C}^{3}\right)$. Then $\mathscr{M}_{\theta}\left(\mathbb{C}^{2}\right)$ is isomorphic to $Y$ by Lemma 1 .

Before stating the proposition, we need some notation. We diagonalize $G$ and write

$$
g=\operatorname{diag}\left(\zeta_{n}^{a_{g}}, \zeta_{n}^{b_{g}}\right)
$$

for $g \in G$ where $\zeta_{n}$ is a primitive $n$-th root of unity. Put

$$
\begin{aligned}
& N_{2}:=\mathbb{Z}^{2}+\sum_{g \in G} \mathbb{Z} \cdot \frac{1}{n}\left(a_{g}, b_{g}\right), \\
& N_{3}:=\mathbb{Z}^{3}+\sum_{g \in G} \mathbb{Z} \cdot \frac{1}{n}\left(a_{g}, b_{g},-a_{g}-b_{g}\right)
\end{aligned}
$$

which are the lattices of one-parameter subgroups for the toric varieties $\mathbb{C}^{2} / G$ and $\mathbb{C}^{3} / G$ respectively. The junior simplex $\Delta \subset\left(N_{3}\right)_{\mathbb{R}}$ is the triangle with vertices $e_{1}, e_{2}, e_{3}$ where $\left\{e_{1}, e_{2}, e_{3}\right\}$ is the basis of $\mathbb{Z}^{3}$ with $e_{1}, e_{2} \in \mathbb{Z}^{2}$. A 
crepant resolution $U$ corresponds to a basic triangulation of $\Delta$. For a basic triangulation $\Sigma$ of $\Delta$, let $U_{\Sigma}$ be the corresponding crepant resolution.

Consider the natural projection

$$
p_{12}: N_{3} \rightarrow N_{2}
$$

and put $\Delta^{\prime}:=p_{12}(\Delta) \cong \Delta$. Let $e_{i}^{\prime} \in\left(\mathbb{R}_{\geq 0}\right) e_{i} \cap N_{2}$ be the primitive vector and write $e_{i}=m_{i} e_{i}^{\prime}$ for $i=1,2$. If $B_{i} \subset \mathbb{C}^{2} / G$ denote the divisor corresponding to $e_{i}^{\prime}$, then

$$
B:=\frac{m_{1}-1}{m_{1}} B_{1}+\frac{m_{2}-1}{m_{2}} B_{2}
$$

is the boundary divisor for the quotient $\mathbb{C}^{2} / G$. A resolution $Y$ of $\mathbb{C}^{2} / G$ is given by choosing primitive vectors $v_{0}, v_{1}, \ldots, v_{s}$ of $\left(\mathbb{Z}_{\geq 0}\right)^{2} \cap N_{2}$ such that $v_{0}=e_{1}^{\prime}, v_{s}=e_{2}^{\prime}$ and $\left\{v_{i-1}, v_{i}\right\}$ is a basis of $N_{2}$ for $i=1, \ldots, s$. If $E_{i}$ denotes the exceptional divisor corresponding to $v_{i}$ for $i=1, \ldots, s-1$, then the discrepancy $a_{i}$ of $E_{i}$ for the pair $(X, B)$ is $\alpha_{i}+\beta_{i}-1$ where $v_{i}=\left(\alpha_{i}, \beta_{i}\right)$. Therefore, $Y$ is dominated by the maximal resolution $Y_{\max }$ of $(X, B)$ if and only if all of $v_{1}, \ldots, v_{s-1}$ are in $\Delta^{\prime}$.

Let $G_{(1,0)} \subset G$ be the stabilizer subgroup of $(1,0) \in \mathbb{C}^{2}=\mathbb{C}^{2} \times\{0\} \subset \mathbb{C}^{3}$. Then $G_{(1,0)}$ acts on $\{1\} \times \mathbb{C}^{2} \cong \mathbb{C}^{2}$ as a subgroup of $\operatorname{SL}(2)$ and the quotient $\left(\{1\} \times \mathbb{C}^{2}\right) / G_{(1,0)}$ is a closed subvariety of $\mathbb{C}^{3} / G$. Let

$$
W \rightarrow\left(\{1\} \times \mathbb{C}^{2}\right) / G_{(1,0)}
$$

be the minimal resolution. Notice that $W$ is contained in any crepant resolution $U$ of $\mathbb{C}^{3} / G$ since $\left(\{1\} \times \mathbb{C}^{2}\right) / G_{(1,0)} \subset \mathbb{C}^{3} / G$ is transversal to the onedimensional stratum $\left(\mathbb{C}^{\times} \times\{(0,0)\}\right) / G$. Now we prove the following proposition. The surjectivity of the ample cones will be used in the proof of the main theorem.

Proposition 2. Let $Y \rightarrow \mathbb{C}^{2} / G$ be a resolution dominated by $Y_{\max }$. Then there is a projective crepant resolution $U=U_{\Sigma} \rightarrow \mathbb{C}^{3} / G$ containing $Y$ such that the restriction map $\operatorname{Amp}(U) \rightarrow \operatorname{Amp}(W)$ of the ample cones is surjective.

Proof. Since $Y$ is dominated by $Y_{\max }$, it is defined by primitive vectors $v_{0}, v_{1}, \ldots, v_{s} \in \Delta^{\prime} \cap N_{2}$. Let $w_{i} \in \Delta \cap N_{3}$ be the unique lift of $v_{i}$. For a basic triangulation $\Sigma$ of $\Delta, U=U_{\Sigma}$ contains $Y$ if and only if the points connected to $e_{3}$ in $\Sigma$ are exactly $w_{0}, \ldots, w_{s}$.

We prove the assertion by the induction on the order $|G|$ of $G$. If $|G|=1$, then there is nothing to prove. We consider the number

$$
v:=\#\left(\left\{w_{0}, \ldots, w_{s-1}\right\} \backslash\left\{e_{1}\right\}\right) \geq 0 .
$$


If $v=0$, then $s$ must be 1 and $w_{0}=e_{1}$ is a primitive vector. Especially, $\left\{e_{1}, v_{1}\right\}$ is a basis of $N_{2}$. In this case, $\Delta$ has a unique basic triangulation $\Sigma$ and $U_{\Sigma} \cong W \times \mathbb{C}$. Hence the restriction map $\operatorname{Amp}\left(U_{\Sigma}\right) \rightarrow \operatorname{Amp}(W)$ is an isomorphism.

Suppose $v>0$. Let $w \in\left\{w_{0}, \ldots, w_{s-1}\right\} \backslash\left\{e_{1}\right\}$ be a point such that the coefficient of $e_{3}$ in $w$ is the smallest. Then $w$ determines a star subdivision of $\Delta: \Delta=\bigcup_{i=1}^{3} \Delta_{i}$ where $\Delta_{1}, \Delta_{2}, \Delta_{3}$ are the triangles $w e_{2} e_{3}, w e_{1} e_{3}, w e_{1} e_{2}$ respectively. Note that either $\Delta_{2}$ or $\Delta_{3}$ may be degenerate, in which case we simply ignore the degenerate one in the sequel. This subdivision of $\Delta$, which is denoted by $\Sigma_{0}$, determines a projective crepant birational morphism $U_{\Sigma_{0}} \rightarrow$ $\mathbb{C}^{3} / G$ where $U_{\Sigma_{0}}$ is a toric variety with at most Gorenstein quotient singularities. The choice of $w$ implies that $w_{0}, \ldots, w_{s}$ are in $\Delta_{1} \cup \Delta_{2}$. Hence by the induction hypothesis, there are basic triangulations $\Sigma_{1}$ and $\Sigma_{2}$ of $\Delta_{1}$ and $\Delta_{2}$ respectively, which satisfy the following conditions: in $\Sigma_{1} \cup \Sigma_{2}$, the vertices connected to $e_{3}$ are exactly $w_{0}, \ldots, w_{s}$, the map $\operatorname{Amp}\left(U_{\Sigma_{1}}\right) \rightarrow \operatorname{Amp}(W)$ is surjective and $\operatorname{Amp}\left(U_{\Sigma_{2}}\right)$ is non-empty. We choose an arbitrary basic triangulation $\Sigma_{3}$ of $\Delta_{3}$ with non-empty $\operatorname{Amp}\left(U_{\Sigma_{3}}\right)$. Combining the triangulations $\Sigma_{1}, \quad \Sigma_{2}$ and $\Sigma_{3}$ together, we obtain a basic triangulation of $\Delta$ such that $U_{\Sigma} \supset Y$. Since $\Delta=\bigcup_{i=1}^{3} \Delta_{i}$ is a star subdivision, we see that $U_{\Sigma} \rightarrow U_{\Sigma_{0}}$ is a projective morphism and the map $\operatorname{Amp}\left(U_{\Sigma}\right) \rightarrow \operatorname{Amp}\left(U_{\Sigma_{1}}\right)$ is surjective. Therefore, the morphism $U_{\Sigma} \rightarrow \mathbb{C}^{3} / G$ is also projective and $\operatorname{Amp}\left(U_{\Sigma}\right) \rightarrow$ $\operatorname{Amp}(W)$ is surjective.

\section{6. $G$-constellations on a variety}

In the case of $G$-constellations for non-abelian $G \subset \mathrm{GL}(2, \mathbb{C})$, we shall use the iterated construction of moduli spaces for a normal subgroup of $G$ as in [IINdC13]. In order to do so, we have to consider $G$-constellations on a variety, rather than an affine space. Especially, the space of stability parameters will be larger than the affine case in general.

Suppose $U$ is a quasi projective variety of finite type over $\mathbb{C}$ and $G$ is a finite group acting on $U$. Let $\operatorname{coh}^{G}(U)$ be the abelian category of $G$ equivariant coherent sheaves on $U$ and $\operatorname{coh}_{\text {cpt }}^{G}(U)$ its subcategory consisting of sheaves whose supports are proper over $\mathbb{C}$. The corresponding Grothendieck groups are denoted by $K\left(\operatorname{coh}^{G}(U)\right)$ and $K\left(\operatorname{coh}_{\text {cpt }}^{G}(U)\right)$ respectively. We also consider the perfect derived category $\operatorname{Perf}^{G}(U)$ of $G$-equivariant perfect complexes and its Grothendieck group $K\left(\operatorname{Perf}^{G}(U)\right)$. For $\alpha \in K\left(\operatorname{Perf}^{G}(U)\right)$ and $\beta \in K\left(\operatorname{coh}_{\mathrm{cpt}}^{G}(U)\right)$, we write

$$
\chi(\alpha, \beta):=\sum_{i}(-1)^{i} \operatorname{dim} \operatorname{Ext}_{\mathcal{O}_{U}}^{i}(\alpha, \beta)^{G} .
$$


Let $\operatorname{coh}_{0 \text {-dim }}^{G}(U)$ be the subcategory of $\operatorname{coh}_{\text {cpt }}^{G}(U)$ consisting of sheaves with 0 -dimensional support. We define the stability condition of objects in $\operatorname{coh}_{0 \text {-dim }}^{G}(U)$.

Definition 4. Fix a class $\xi \in K\left(\operatorname{Perf}^{G}(U)\right)$. An object $E \in \operatorname{coh}_{0 \text {-dim }}^{G}(U)$ is said to be $\xi$-stable (or $\xi$-semistable) if $\chi(\xi, E)=0$ and if for every non-trivial $G$-equivariant subsheaf $F$ of $E, \chi(\xi,[F])>0$ (or $\chi(\xi,[F]) \geq 0$ ).

In the case where $U=\mathbb{C}^{N}$ is an affine space with a linear $G$-action, $K\left(\operatorname{Perf}^{G}(U)\right)=K\left(\operatorname{coh}^{G}(U)\right)$ is isomorphic to (the dual of) the representation ring $R(G)$ and the definition coincides with the (ZZZ-valued) one in $\S 2.1$.

We have a well-defined function rank $: K\left(\operatorname{Perf}^{G}(U)\right) \rightarrow \mathbb{Z}$ which extends the rank of a locally free sheaf. Put

$$
K\left(\operatorname{Perf}^{G}(U)\right)^{0}:=\left\{\xi \in K\left(\operatorname{Perf}^{G}(U)\right) \mid \operatorname{rank} \xi=0\right\} .
$$

Definition 5. A $G$-constellation on $U$ is a $G$-equivariant coherent sheaf $E$ on $U$ with finite support such that $H^{0}(E)$ is isomorphic to the regular representation of $G$ as a representation of $G$ and $\chi(\xi, E)=0$ for any $\xi \in$ $K\left(\operatorname{Perf}^{G}(U)\right)^{0}$.

For any $\xi \in K\left(\operatorname{Perf}^{G}(U)\right)^{0}$, we can discuss the $\xi$-(semi)stabilities of $G$-constellations on $U$ according to Definition 4. Since the multiplication by a positive integer does not change the stability condition, we may replace $K\left(\operatorname{Perf}^{G}(U)\right)^{0}$ by $K\left(\operatorname{Perf}^{G}(U)\right)_{\mathbb{Q}}^{0}$.

REMARK 1. In general, there may exist an object $E$ supported on several fixed points such that $H^{0}(E) \cong R(G)$ but $\chi(\xi, E) \neq 0$ for some $\xi \in$ $K\left(\operatorname{Perf}^{G}(U)\right)^{0}$. Definition 5 excludes such cases.

REMARK 2. If $U$ is smooth, then $K\left(\operatorname{Perf}^{G}(U)\right)$ coincides with $K\left(\operatorname{coh}^{G}(U)\right)$ and we write $K\left(\operatorname{coh}^{G}(U)\right)^{0}$ instead of $K\left(\operatorname{Perf}^{G}(U)\right)^{0}$.

Now we define the moduli functors of $G$-constellations:

Definition 6. Fix a class $\xi \in K\left(\operatorname{Perf}^{G}(U)\right)_{\mathbb{Q}}^{0}$. Then the moduli functor for the $\xi$-stable $G$-constellations on $U$ is defined to be the functor

$S \mapsto\{$ flat families of $\xi$-stable $G$-constellations parameterized by $S\} / \sim$ for a locally noetherian scheme $S$ over $\mathbb{C}$ where $E_{S} \sim F_{S}$ for flat families $E_{S}$ and $F_{S}$ means that there is a line bundle $L$ on $S$ such that $E_{S} \cong F_{S} \otimes L$.

REMARK 3. We show the existence of the moduli scheme in a very special case in Theorem 6. We do not discuss the existence problem in a general case in this paper. 


\section{Iterated construction of moduli spaces}

In this section, let $V$ denote either $\mathbb{C}^{2}$ or $\mathbb{C}^{3}$ and consider a finite subgroup $G \subset \mathrm{GL}(V)$ with a normal subgroup $N$ of $G$ such that $N \subset \operatorname{SL}(V)$. Let

$$
\theta^{N}: R(N) \rightarrow \mathbb{Z}
$$

be a generic stability parameter for $N$-constellations on $V$, which is fixed by the conjugate action of $G$ on $R(N)$. Put $Y_{N}=\mathscr{M}_{\theta^{N}}(V)$ and $\bar{G}=G / N$. Since $N \subset \operatorname{SL}(V)$ and $\operatorname{dim} V \leq 3$, there is an equivalence

$$
\Phi: D^{b}\left(\operatorname{coh}^{\bar{G}}\left(Y_{N}\right)\right) \cong D^{b}\left(\operatorname{coh}^{G}(V)\right)
$$

as in [IU15, Theorem 4.1] defined by

$$
\Phi(-)=\mathbb{R}\left(p_{V}\right)_{*}\left(\left(p_{Y_{N}}\right)^{*}(-) \otimes \mathscr{U}\right)
$$

where $p_{V}, p_{Y_{N}}$ are the projections of $Y_{N} \times V$ and $\mathscr{U}$ is the universal family of $N$-constellations.

Lemma 2. Let $\mathscr{E}$ be a $\bar{G}$-equivariant coherent sheaf on $Y_{N}$ with finite support. Then $\mathscr{E}$ is a $\bar{G}$-constellation on $Y_{N}$ if and only if $\Phi(\mathscr{E})$ is a $G$-constellation on $V$. In this case, $\Phi(\mathscr{E})$ is $\theta^{N}$-semistable.

Proof. By the definition of $\Phi$, we can see that $\Phi(\mathscr{E})$ is a 0 -dimensional sheaf. Since $\Phi$ is an equivalence, we have $\chi(\xi, \mathscr{E})=\chi(\Phi(\xi), \Phi(\mathscr{E}))$. Moreover, we can see rank $\xi=\operatorname{rank} \Phi(\xi)$ for any $\xi \in K\left(\operatorname{coh}^{\bar{G}}\left(Y_{N}\right)\right)$. Therefore, if $\mathscr{E}$ is a $\bar{G}$-constellation, $\chi(\xi, \Phi(\mathscr{E}))=0$ for any $\xi \in K\left(\operatorname{coh}^{G}(V)\right)^{0}$. This implies that $H^{0}(\Phi(\mathscr{E}))$ is a multiple of the regular representation $\mathbb{C}[G]$. If we regard $\mathscr{E}$ as an object of $\operatorname{coh}\left(Y_{N}\right)$, it is an Artinian sheaf of length $|\bar{G}|$ and therefore $\Phi(\mathscr{E})$ as an object of $\operatorname{coh}^{N}(V)$ has a filtration of length $|\bar{G}|$ whose factors are $\theta^{N}$-stable $N$-constellations. Therefore, $\Phi(\mathscr{E})$ is $\theta^{N}$-semistable and $H^{0}(\Phi(\mathscr{E}))$ as a representation of $N$ is the direct sum of $|\bar{G}|$ copies of the regular representation of $N$. This implies that $H^{0}(\Phi(\mathscr{E})) \cong \mathbb{C}[G]$ and therefore $\Phi(\mathscr{E})$ is a $G$-constellation. The converse is proved in the same way.

The following lemma follows from the arguments in [BKR01, §8]:

Lemma 3. Let $E$ be an $N$-equivariant coherent sheaf on $V$ with finite support such that $H^{0}(E)$ is isomorphic to $\mathbb{C}[N]^{\oplus s}$ for some integer $s>0$ as a $\mathbb{C}[N]$ module. If $E$ is $\theta^{N}$-stable, then we have $s=1$, i.e., $E$ is an $N$-constellation.

We compose $\theta^{N}$ with the restriction map $R(G) \rightarrow R(N)$ and regard it as a stability parameter for $G$-constellations as in [IINdC13, §2.2]. 
Lemma 4. Let $E$ be a G-equivariant coherent sheaf on $V$ with finite support such that $H^{0}(E) \cong \mathbb{Z}[G]^{\oplus s}$ for some $s$. If $E$ is $\theta^{N}$-semistable in $\operatorname{coh}^{G}(V)$, then it is also $\theta^{N}$-semistable in $\operatorname{coh}^{N}(V)$.

Proof. Let $\eta: R(N) \rightarrow \mathbb{Z}$ be a group homomorphism such that $\eta(\rho)>0$ for any irreducible representation $\rho$ of $N$. We further suppose $\eta$ is invariant under the conjugate action of $G$. Then,

$$
Z(E):=\theta^{N}\left(H^{0}(E)\right)+\sqrt{-1} \eta\left(H^{0}(E)\right)
$$

defines a $G$-invariant Bridgeland stability condition [Bri07, Example 5.5] (see also [BCZ17, Lemma 7.1.3]) on $\operatorname{coh}^{N}(V)_{0}$, the category of $N$-equivariant coherent sheaves on $V$ with 0-dimensional support. As in [BCZ17, Lemma 7.1.5], the equality $\theta^{N}\left(H^{0}(E)\right)=0$ implies that $E$ is $\theta^{N}$-semistable if and only if it is semistable with respect to $Z$. Assume $E$ is not $\theta^{N}$-semistable and let $F \subset E$ be the first step of the Harder-Narasimhan filtration of $E$ in $\operatorname{coh}^{N}(E)$ with respect to $Z$. Then the uniqueness of the HN filtration and the $G$-invariance of $Z$ imply that $F$ is invariant under the $G$-action. This means that $F$ is a subsheaf of $E$ in $\operatorname{coh}^{G}(V)$, which contradicts the $\theta^{N}$-semistability of $E$ in $\operatorname{coh}^{G}(V)$.

Proposition 3. The functor $\Phi$ induces a bijection from the set of $\bar{G}$-constellations on $Y_{N}$ to the set of $\theta^{N}$-semistable G-constellations on $V$.

Proof. If $\mathscr{E}$ is a $\bar{G}$-constellation on $Y_{N}$, then $\Phi(\mathscr{E})$ is a $\theta^{N}$-semistable $G$-constellation by Lemma 2. Conversely, suppose $E$ is a $\theta^{N}$-semistable $G$-constellation on $V$. By Lemma 2, it suffices to show that $\Phi^{-1}(E)$ lies in $\operatorname{coh}^{\bar{G}}\left(Y_{N}\right)$ and has a 0 -dimensional support. For this purpose, we may regard $\Phi$ as an equivalence $D^{b}\left(\operatorname{coh} Y_{N}\right) \cong D^{b}\left(\operatorname{coh}^{N}(V)\right)$. By Lemma $4, E$ is $\theta^{N}$. semistable as a sheaf in $\operatorname{coh}^{N}(V)$ and therefore has a filtration whose factors are $\theta^{N}$-stable $N$-constellations by Lemma 3. Then, $\Phi^{-1}(E)$ as an object in $D^{b}\left(\operatorname{coh}\left(Y_{N}\right)\right)$ is a sheaf with a filtration whose factors are skyscraper sheaves. This is what we needed.

Let

$$
\varphi: K\left(\operatorname{coh}^{\bar{G}}\left(Y_{N}\right)\right)_{\mathbb{Q}}^{0} \stackrel{\sim}{\rightarrow} K\left(\operatorname{coh}^{G}(V)\right)_{\mathbb{Q}}^{0} \cong \Theta
$$

be the isomorphism induced by $\Phi$. The following theorem generalizes [IINdC13, Theorem 2.6].

THEOREM 6. Let $\theta^{N}: R(N) \rightarrow \mathbb{Z}$ be a generic stability condition for $N$-constellations fixed by the conjugate action of $G$ and $\xi \in K\left(\operatorname{coh}^{\bar{G}}\left(Y_{N}\right)\right)^{0}$ be a stability parameter for $\bar{G}$-constellations on $Y_{N}$. 
(1) There exists a scheme $\mathscr{M}_{\xi}\left(Y_{N}\right)$ representing the moduli functor for $\xi$-stable $\bar{G}$-constellations on $Y_{N}$.

(2) If we put

$$
\theta:=m \theta^{N}+\varphi(\xi)
$$

for $m \gg 0$, then $\mathscr{M}_{\theta}(V)$ is isomorphic to the moduli space $\mathscr{M}_{\xi}\left(Y_{N}\right)$ of $\xi$-stable $\bar{G}$-constellations on $Y_{N}$.

Proof. What we prove is that $\mathscr{M}_{\theta}(V)$ in (2) represents the moduli functor in (1). We choose $m$ so that

$$
m>\sum_{\rho \in \operatorname{Irr}(G)}|(\varphi(\xi))(\rho)| \operatorname{dim} \rho .
$$

Then for any subsheaf $F$ of a $G$-constellation, we have $|(\varphi(\xi))(F)|<m$.

Let $\mathscr{E}$ be a $\xi$-stable $\bar{G}$-constellation on $Y_{N}$. Then $\Phi(\mathscr{E})$ is a $\theta^{N}$-semistable $G$-constellation by Proposition 3. Therefore, a subsheaf $F$ of $\Phi(\mathscr{E})$ satisfies $\theta^{N}(F) \geq 0$. If $\theta^{N}(F)>0$, then we have $\theta(F)>0$ by our choice of $m$. If $\theta^{N}(F)=0$, then there is a subsheaf $\mathscr{F}$ of $\mathscr{E}$ such that $F=\Phi(\mathscr{F})$ as in [IINdC13, Lemma 2.6]. Then we obtain $\theta(F)=\chi(\xi, \mathscr{F})>0$ by the $\xi$-stability of $\mathscr{E}$. Thus $\Phi(\mathscr{E})$ is $\theta$-stable.

Conversely, suppose $E$ is a $\theta$-stable $G$-constellation on $V$. Then it is $\theta^{N}$ semistable by our choice of $m$ and therefore $\mathscr{E}:=\Phi^{-1}(E)$ is a $\bar{G}$-constellation by Proposition 3. For a subsheaf $\mathscr{F} \subset \mathscr{E}, F:=\Phi(\mathscr{F})$ has a filtration as an object of $\operatorname{coh}^{N}(V)$ whose factors are $N$-constellations. Therefore $F$ satisfies $\theta^{N}(F)=0$ and hence we obtain $\chi(\xi, \mathscr{F})=\theta(F)>0$, which proves the $\xi$-stability of $\mathscr{F}$.

Thus we have a bijection between $\xi$-stable $\bar{G}$-constellations and $\theta$-stable $G$-constellations. To establish an isomorphism $\mathscr{M}_{\theta}(V) \cong \mathscr{M}_{\xi}\left(Y_{N}\right)$, we show that for any locally noetherian scheme $S$ over $\mathbb{C}$, this bijection can be extended to a bijection between flat families of $\xi$-stable $\bar{G}$-constellations and flat families of $\theta$-stable $G$-constellations parameterized by $S$. Let $\mathscr{U}$ be the universal $N$-constellation on $Y_{N} \times V$ and $\mathscr{U}_{S}$ be the pull back of $\mathscr{U}$ to $Y_{N} \times V \times S$. Then we can define a functor

$$
\Phi_{S}: D^{b}\left(\operatorname{coh}^{\bar{G}} Y_{N} \times S\right) \rightarrow D^{b}\left(\operatorname{coh}^{G} V \times S\right)
$$

by

$$
\Phi_{S}(-)=\mathbb{R}\left(p_{V \times S}\right)_{*}\left(\mathscr{U}_{S} \otimes p_{Y_{N} \times S}^{*}(-)\right)
$$

whose quasi-inverse is given by

$$
\Phi_{S}^{-1}(-)=\left(\left(p_{Y_{N} \times S}\right)_{*}\left(\mathscr{U}_{S}^{\vee}[\operatorname{dim} V] \stackrel{\mathbb{L}}{\otimes} p_{V \times S}^{*}(-)\right)^{N} .\right.
$$


Suppose $\mathscr{E}_{S}$ is a flat family of $\xi$-stable $\bar{G}$-constellations on $Y_{N}$ parameterized by $S$. Then, for any geometric point $s$ of $S$, we have $\Phi_{S}\left(\mathscr{E}_{S}\right) \stackrel{\mathbb{L}}{\otimes} \mathcal{O}_{S} \cong \Phi\left(\mathscr{E}_{S}\right)$ as in [Bri99, Lemma 4.1], which is a $\theta$-stable $G$-constellation on $V$. Hence the argument in [Bri99, Proposition 4.2] implies that $\Phi_{S}\left(\mathscr{E}_{S}\right)$ is actually a flat family of $G$-constellations on $V$. Conversely, if $E_{S}$ is a flat family of $\theta$-stable $G$-constellations, the same argument shows that $\Phi_{S}^{-1}\left(E_{S}\right)$ is a flat family of $\xi$-stable $N$-constellations on $Y_{N}$.

\section{The case $G \ni-I$}

In this section, put $V=\mathbb{C}^{2}$ and assume that $G \subset \mathrm{GL}(V)$ contains $-I$, where $I$ is the identity matrix. We put $N:=\langle-I\rangle \subset G$ and $\bar{G}:=G / N$. Let $\theta^{N}$ be any generic stability parameter for $N$-constellations (which is automatically fixed by the conjugate action of $G$ since $N$ is central) and let $Y_{N}=$ $\mathscr{M}_{\theta^{N}}(V)$ be the moduli space of $N$-constellations on $V$, on which $\bar{G}$ acts naturally. Since $Y_{N}$ is a crepant resolution of the $A_{1}$ singularity $V / N$, the maximal resolution of $\left(Y_{N} / \bar{G}, B_{N}\right)$ coincides with the maximal resolution of $(X, B)$, where $B_{N}$ is the boundary divisor on $Y_{N}$ determined by the ramification of $Y_{N} \rightarrow Y_{N} / \bar{G}$.

Let $C$ be the exceptional curve of $Y_{N} \rightarrow V / N$. Then the equivalence (7.1) restricts to the equivalence

$$
\Phi: D^{b}\left(\operatorname{coh}_{C}^{\bar{G}}\left(Y_{N}\right)\right) \cong D^{b}\left(\operatorname{coh}_{0}^{G}(V)\right)
$$

of full subcategories consisting of objects supported by the subsets $C \subset Y_{N}$ and $\{0\} \subset V$ respectively. Consider the Grothendieck groups of (8.1):

$$
K\left(\operatorname{coh}_{C}^{\bar{G}}\left(Y_{N}\right)\right) \cong K\left(\operatorname{coh}_{0}^{G}(V)\right)
$$

where $K\left(\operatorname{coh}_{0}^{G}(V)\right)$ is isomorphic to the representation ring $R(G)$ of $G$. Recall that there is a perfect pairing

$$
\chi: K\left(\operatorname{coh}^{G}(V)\right) \times K\left(\operatorname{coh}_{0}^{G}(V)\right) \rightarrow \mathbb{Z}
$$

defined by (6.1), which is isomorphic to

$$
\chi: K\left(\operatorname{coh}^{\bar{G}}\left(Y_{N}\right)\right) \times K\left(\operatorname{coh}_{C}^{\bar{G}}\left(Y_{N}\right)\right) \rightarrow \mathbb{Z}
$$

by $\Phi$. Let

$$
F_{i} K\left(\operatorname{coh}_{C}^{\bar{G}}\left(Y_{N}\right)\right) \subset K\left(\operatorname{coh}_{C}^{\bar{G}}\left(Y_{N}\right)\right)
$$

be the subgroup generated by the classes of objects whose supports are at most $i$-dimensional. Then the classes of $\bar{G}$-constellations on $Y_{N}$ lie in 
$F_{0} K\left(\operatorname{coh}_{C}^{\bar{G}}\left(Y_{N}\right)\right)$ and for a stability parameter

$$
\xi \in K\left(\operatorname{coh}^{\bar{G}}\left(Y_{N}\right)\right)_{\mathbb{Q}} \cong K\left(\operatorname{coh}_{C}^{\bar{G}}\left(Y_{N}\right)\right)_{\mathbb{Q}}^{*}
$$

the actual stability condition depends only on its image in $F_{0} K\left(\operatorname{coh}_{C}^{\bar{G}}\left(Y_{N}\right)\right)_{\mathbb{Q}}^{*}$. In the next subsection, we investigate the structure of $F_{0} K\left(\operatorname{coh}_{C}^{\bar{G}}\left(Y_{N}\right)\right)$.

8.1. Structure of $F_{0} K\left(\operatorname{coh}_{C}^{\bar{G}}\left(Y_{N}\right)\right)$. In this subsection, we assume that $G$ is not abelian. Notice that $G$ acts on the exceptional curve $C \cong \mathbb{P}(V)$ through the homomorphism

$$
G \hookrightarrow \mathrm{GL}(V) \rightarrow \operatorname{PGL}(V)
$$

and let $Z \subset G$ be the kernel of $G \rightarrow \operatorname{PGL}(V)$. It is the subgroup consisting of scalar matrices in $G$.

Since $G$ is non-abelian, $G / Z \subset \operatorname{PGL}(V)$ is a polyhedral (or dihedral) group acting on $\mathbb{P}(V)$ which we regard as a (real) 2-sphere. There are three nonfree $G / Z$-orbits in $C$ : the projections of the vertices, edges and faces of the regular polyhedron to the sphere. These orbits are denoted by $O_{1}, O_{2}$ and $O_{3}$ respectively.

For a $\bar{G}$-orbit $O \subset C$, let $\operatorname{coh}_{O}^{\bar{G}}\left(Y_{N}\right)$ denote the category of $\bar{G}$-equivariant coherent sheaves supported on $O$. Then we have an equivalence

$$
\operatorname{coh}_{O}^{\bar{G}}\left(Y_{N}\right) \cong \operatorname{coh}_{P}^{\bar{G}_{P}}\left(Y_{N}\right)
$$

where $\bar{G}_{P}$ is the stabilizer subgroup of a point $P \in O$ and $\operatorname{coh}_{P}^{\bar{G}_{P}}\left(Y_{N}\right)$ is the category of $\bar{G}_{P}$-equivariant coherent sheaves supported on $P$. Taking the Grothendieck groups of the both sides, we obtain

$$
K\left(\operatorname{coh}_{O}^{\bar{G}}\left(Y_{N}\right)\right) \cong R\left(\bar{G}_{P}\right)
$$

where $R\left(\bar{G}_{P}\right)$ is the representation ring of $\bar{G}_{P}$ regarded as an additive group.

Let $\bar{G}_{k} \subset \bar{G}$ be the stabilizer subgroup of a point in $O_{k}$, which is an abelian group since $\bar{Z}:=Z / N \subset \bar{G}_{k}$ is central and $\bar{G}_{k} / \bar{Z}$ is cyclic. We consider the pushforward maps

$$
K\left(\operatorname{coh}_{O_{k}}^{\bar{G}}\left(Y_{N}\right)\right) \rightarrow F_{0} K\left(\operatorname{coh}_{C}^{\bar{G}}\left(Y_{N}\right)\right)
$$

for $k=1,2,3$. By (8.4) for $O=O_{k}$, these maps are regarded as maps

$$
\beta_{k}: R\left(\bar{G}_{k}\right) \rightarrow F_{0} K\left(\operatorname{coh}_{C}^{\bar{G}}\left(Y_{N}\right)\right) .
$$

Since $\bar{Z}$ is a subgroup of $\bar{G}_{k}$, we have the induction maps

$$
\alpha_{k}: R(\bar{Z}) \rightarrow R\left(\bar{G}_{k}\right)
$$


Define a map $\alpha: R(\bar{Z})^{\oplus 2} \rightarrow R\left(\bar{G}_{1}\right) \oplus R\left(\bar{G}_{2}\right) \oplus R\left(\bar{G}_{3}\right)$ by

$$
\alpha(a, b)=\left(\alpha_{1}(a),-\alpha_{2}(a)+\alpha_{2}(b),-\alpha_{3}(b)\right) .
$$

The purpose of this subsection is to prove the following.

Proposition 4. Let $\bar{G}_{k}, \beta_{k}$, $\alpha$ be as above. Then the following is an exact sequence of additive groups:

$$
0 \rightarrow R(\bar{Z})^{\oplus 2} \stackrel{\alpha}{\rightarrow} R\left(\bar{G}_{1}\right) \oplus R\left(\bar{G}_{2}\right) \oplus R\left(\bar{G}_{3}\right) \stackrel{\beta}{\rightarrow} F_{0} K\left(\operatorname{coh}_{C}^{\bar{G}}\left(Y_{N}\right)\right) \rightarrow 0
$$

where $\beta=\left(\beta_{1}, \beta_{2}, \beta_{3}\right)$.

The proof of the proposition is divided into three steps below. We first show that $\beta$ is surjective:

STEP 1. The additive group $F_{0} K\left(\operatorname{coh}_{C}^{\bar{G}}\left(Y_{N}\right)\right)$ is generated by sheaves supported on $\mathrm{O}_{1} \cup \mathrm{O}_{2} \cup \mathrm{O}_{3}$.

Proof. It is obvious that $F_{0} K\left(\operatorname{coh}_{C}^{\bar{G}}\left(Y_{N}\right)\right)$ is generated by simple objects (objects having no non-trivial subobjects). Moreover, a simple object is supported on a single orbit $O$ and is determined by an irreducible representation of the stabilizer subgroup $\bar{G}_{P}$ of a point $P \in O$ by (8.3). Therefore, it is sufficient to show that the class in $K\left(\operatorname{coh}_{C}^{\bar{G}}\left(Y_{N}\right)\right)$ of a simple object $\mathscr{E}$ supported on a free $G / Z$-orbit $O_{f}$ coincides with the class of some object $\mathscr{F}$ supported on $O_{1} \cup O_{2} \cup O_{3}$. Actually, we prove that for any $k \in\{1,2,3\}$ we can choose such an object $\mathscr{F}$ supported on $O_{k}$. Simple objects supported on the orbit $O_{f}$ are determined by irreducible representations of the stabilizer subgroup $\bar{Z} \subset \bar{G}$ by (8.3). To describe them, notice that $C=\mathbb{P}(V)$ carries a $G$-equivariant line bundle $\mathscr{L}=\mathcal{O}_{C}(1)$ on which an element $\lambda I \in Z$ acts as the fiber-wise scalar multiplication by $\lambda$. On $\mathscr{L}^{2}$, the $G$-action is reduced to a $\bar{G}$-action and the induced actions of $\bar{Z}$ on the fibers of $\mathscr{L}^{0}, \mathscr{L}^{2}, \ldots, \mathscr{L}^{2(l-1)}$ are the irreducible representations of the cyclic group $\bar{Z}$, where $l$ is the order of $\bar{Z}$. Therefore, the simple objects supported on $O_{f}$ are

$$
\left.\mathscr{L}^{0}\right|_{O_{f}},\left.\mathscr{L}^{2}\right|_{O_{f}}, \ldots,\left.\mathscr{L}^{2(l-1)}\right|_{O_{f}},
$$

where we regard $O_{f}$ as a reduced subscheme. Now consider the exact sequences

$$
\left.0 \rightarrow \mathscr{L}^{2 i} \otimes \mathcal{O}_{C}\left(-O_{f}\right) \rightarrow \mathscr{L}^{2 i} \rightarrow \mathscr{L}^{2 i}\right|_{O_{f}} \rightarrow 0
$$

and

$$
\left.0 \rightarrow \mathscr{L}^{2 i} \otimes \mathcal{O}_{C}\left(-n_{k} O_{k}\right) \rightarrow \mathscr{L}^{2 i} \rightarrow \mathscr{L}^{2 i}\right|_{n_{k} O_{k}} \rightarrow 0
$$


for any $k \in\{1,2,3\}$ where $n_{k}$ is the order of $\bar{G}_{k} / \bar{Z}$. If we show $\mathcal{O}_{C}\left(-O_{f}\right) \cong$ $\mathcal{O}_{C}\left(-n_{k} O_{k}\right)$ in $\operatorname{coh}^{\bar{G}}\left(Y_{N}\right)$, then we obtain

$$
\left[\left.\mathscr{L}^{2 i}\right|_{O_{f}}\right]=\left[\left.\mathscr{L}^{2 i}\right|_{n_{k} O_{k}}\right]
$$

in $K\left(\operatorname{coh}_{C}^{\bar{G}}\left(Y_{N}\right)\right)$ for any $k$ as desired.

Finally, we show $\mathcal{O}_{C}\left(-O_{f}\right) \cong \mathcal{O}_{C}\left(-n_{k} O_{k}\right)$. Let $\bar{C} \cong \mathbb{P}^{1}$ be the quotient of $C$ by the action of $G / Z$. Then both $\mathcal{O}_{C}\left(-O_{f}\right)$ and $\mathcal{O}_{C}\left(-n_{k} O_{k}\right)$ are the pullbacks of $\mathcal{O}_{\bar{C}}(-1)$ (equipped with the trivial $\bar{G}$-action) and hence we obtain the isomorphism.

STEP 2. $\beta \circ \alpha=0$.

Proof. This is equivalent to the equality

$$
\beta_{1} \circ \alpha_{1}=\beta_{2} \circ \alpha_{2}=\beta_{3} \circ \alpha_{3} \text {. }
$$

We recall the isomorphism (8.4) for a free $G / Z$-orbit $O_{f} \subset C$ :

$$
R(\bar{Z}) \cong K\left(\operatorname{coh}_{O_{f}}^{\bar{G}}\left(Y_{N}\right)\right) .
$$

Then it is sufficient to prove that $\beta_{k} \circ \alpha_{k}$ is identified with the pushforward map

$$
K\left(\operatorname{coh}_{O_{f}}^{\bar{G}}\left(Y_{N}\right)\right) \rightarrow F_{0} K\left(\operatorname{coh}_{C}^{\bar{G}}\left(Y_{N}\right)\right) .
$$

Recall that $K\left(\operatorname{coh}_{O_{f}}^{\bar{G}}\left(Y_{N}\right)\right)$ has a basis of the form (8.6) and that their images in $K\left(\operatorname{coh}_{C}^{\bar{G}}\left(Y_{N}\right)\right)$ satisfy $(8.7)$. Hence the problem is reduced to showing that the map

$$
K\left(\operatorname{coh}_{O_{f}}^{\bar{G}}\left(Y_{N}\right)\right) \rightarrow K\left(\operatorname{coh}_{O_{k}}^{\bar{G}}\left(Y_{N}\right)\right)
$$

defined by

$$
\left[\left.\mathscr{L}^{2 i}\right|_{O_{f}}\right] \mapsto\left[\left.\mathscr{L}^{2 i}\right|_{n_{k} O_{k}}\right]
$$

is identified with the induction map $\alpha_{k}$. The irreducible representation $\rho_{i}$ of $\bar{Z}$ corresponding to $\left[\left.\mathscr{L}^{2 i}\right|_{O_{f}}\right]$ is defined by sending $[\lambda I] \in \bar{Z}$ to $\lambda^{2 i} \in \mathbb{C}^{\times}$. On the other hand, we have

$$
\left[\left.\mathscr{L}^{2 i}\right|_{n_{k} O_{k}}\right]=\sum_{j=0}^{n_{k}-1}\left[\left.\mathscr{L}^{2 i}\left(-j O_{k}\right)\right|_{O_{k}}\right] .
$$

Here $\left.\mathscr{L}^{2 i}\right|_{O_{k}}$ corresponds to a representation of $\bar{G}_{k}$ whose restriction to $\bar{Z}$ is $\rho_{i}$. Moreover, $\left.\mathcal{O}_{C}\left(-j O_{k}\right)\right|_{O_{k}}\left(0 \leq j \leq n_{k}-1\right)$ correspond to the irreducible representations of the cyclic group $\bar{G}_{k} / \bar{Z}$. Thus the element of $R\left(\bar{G}_{k}\right)$ corresponding to $\left[\left.\mathscr{L}^{2 i}\right|_{n_{k} O_{k}}\right]$ is the sum of all the irreducible representations of $\bar{G}_{k}$ whose restrictions to $\bar{Z}$ are $\rho_{i}$. Since $\bar{G}_{k}$ is an abelian group, this is the induced representation of $\rho_{i}$. Thus we obtain $\beta \circ \alpha=0$.

STEP 3. $\operatorname{ker} \beta=\operatorname{Im} \alpha$. 
Proof. Notice that coker $\alpha$ is torsion free, $\beta$ is surjective and $\beta \circ \alpha=0$. Therefore it suffices to show

$$
\operatorname{rank} F_{0} K\left(\operatorname{coh}_{C}^{\bar{G}}\left(Y_{N}\right)\right)=\sum_{k=1}^{3} \operatorname{rank} R\left(\bar{G}_{k}\right)-2 \operatorname{rank} R(\bar{Z}) .
$$

This follows from the following two equalities:

$$
\begin{aligned}
\operatorname{rank} F_{0} K\left(\operatorname{coh}_{C}^{\bar{G}}\left(Y_{N}\right)\right) & =\operatorname{rank} R(G)-\operatorname{rank} R(\bar{Z}) \\
\sum_{k=1}^{3} \operatorname{rank} R\left(\bar{G}_{k}\right) & =\operatorname{rank} R(G)+\operatorname{rank} R(\bar{Z}) .
\end{aligned}
$$

We first consider (8.8). The isomorphism (8.2) reduces (8.8) to the equality

$$
\operatorname{rank} K\left(\operatorname{coh}_{C}^{\bar{G}}\left(Y_{N}\right)\right) / F_{0} K\left(\operatorname{coh}_{C}^{\bar{G}}\left(Y_{N}\right)\right)=\operatorname{rank} R(\bar{Z})
$$

and therefore it suffices to show that the classes

$$
\left[\mathcal{O}_{C}\right],\left[\mathscr{L}^{2}\right], \ldots,\left[\mathscr{L}^{2(l-1)}\right]
$$

form a free basis of the quotient $K\left(\operatorname{coh}_{C}^{\bar{G}}\left(Y_{N}\right)\right) / F_{0} K\left(\operatorname{coh}_{C}^{\bar{G}}\left(Y_{N}\right)\right)$ where

$$
l:=\operatorname{rank} R(\bar{Z})=|\bar{Z}| \text {. }
$$

Recall that $\mathscr{L}^{2} \cong \omega_{C}^{-1}$ is a $\bar{G}$-equivariant line bundle on $C=\mathbb{P}(V)$. Since $\bar{Z}$ acts on $C$ trivially, if we regard $\mathscr{L}^{2}$ as an object of $\operatorname{coh}^{\bar{Z}}(C)$, we have

$$
\mathscr{L}^{2 i} \cong \mathcal{O}_{C}(2 i) \otimes \rho_{\bar{i}} \quad \text { in } \operatorname{coh}^{\bar{Z}}(C)
$$

where $\bar{\imath}=i \bmod l$ and $\rho_{0}, \rho_{1}, \ldots, \rho_{l-1}$ are the irreducible representations of the cyclic group $\bar{Z} \cong \mathbb{Z} / l \mathbb{Z}$. This implies that (8.10) is linearly independent. To see that $(8.10)$ is a generator, we show that for any object $\mathscr{E} \in \operatorname{coh}_{C}^{\bar{G}}\left(Y_{N}\right)$ its class $[\mathscr{E}]$ is a linear combination of $(8.10)$ modulo $F_{0} K\left(\operatorname{coh}_{C}^{\bar{G}}\left(Y_{N}\right)\right)$. We may assume that $\mathscr{E}$ is a locally free sheaf on $C$ and we use the induction on rank $\mathscr{E}$. If rank $\mathscr{E}=0$, there is nothing to prove and we may suppose rank $\mathscr{E}>0$. If we regard $\mathscr{E}$ as an object of $\operatorname{coh}^{\bar{Z}}(C)$, it splits as $\mathscr{E}=\bigoplus_{i} \mathscr{E}_{i} \otimes_{\mathbb{C}} \rho_{i}$ with $\mathscr{E}_{i} \in \operatorname{coh}(C)$. Suppose $\mathscr{E}_{i} \neq 0$. For any integer $m$ we have

$$
\operatorname{Hom}_{\mathcal{O}_{C}}\left(\mathscr{L}^{2 i}, \mathscr{E} \otimes \mathscr{L}^{2 l m}\right)^{\bar{G}}=H^{0}\left(\left(\mathscr{E} \otimes \mathscr{L}^{2 m l-2 i}\right)^{\bar{Z}}\right)^{\bar{G} / \bar{Z}} .
$$

Here, (8.11) shows

$$
\left(\mathscr{E} \otimes \mathscr{L}^{2 m l-2 i}\right)^{\bar{Z}} \cong \mathscr{E}_{i} \otimes \mathcal{O}(2 m l-2 i) \neq 0
$$

and the restriction map

$$
H^{0}\left(\left(\mathscr{E} \otimes \mathscr{L}^{2 m l-2 i}\right)^{\bar{Z}}\right) \rightarrow H^{0}\left(\left.\left(\mathscr{E} \otimes \mathscr{L}^{2 m l-2 i}\right)^{\bar{Z}}\right|_{O_{f}}\right)
$$


is surjective for a $\bar{G} / \bar{Z}$-free orbit $O_{f} \subset C$ if $m$ is sufficiently large. Since $H^{0}\left(\left.\left(\mathscr{E} \otimes \mathscr{L}^{2 m l+2 i}\right)^{\bar{Z}}\right|_{O_{f}}\right)$ is a non-zero multiple of the regular representation of $\bar{G} / \bar{Z}$, its $\bar{G} / \bar{Z}$-invariant part is non-zero. Therefore, $(8.12)$ is non-zero and hence there is a non-zero homomorphism

$$
\alpha: \mathscr{L}^{2 i} \hookrightarrow \mathscr{E} \otimes \mathscr{L}^{2 l m} .
$$

Now the induction hypothesis shows that coker $\alpha$ is a linear combination of (8.10) modulo $F_{0} K\left(\operatorname{coh}_{C}^{\bar{G}}\left(Y_{N}\right)\right)$. This shows that the class $\left[\mathscr{E} \otimes \mathscr{L}^{2 l m}\right]$ is also a linear combination of $(8.10)$ modulo $F_{0} K\left(\operatorname{coh}_{C}^{\bar{G}}\left(Y_{N}\right)\right)$. Since we have

$$
[\mathscr{E}]-\left[\mathscr{E} \otimes \mathscr{L}^{2 l m}\right] \in F_{0} K\left(\operatorname{coh}_{C}^{\bar{G}}\left(Y_{N}\right)\right),
$$

$[\mathscr{E}]$ is a linear combination of (8.10) modulo $F_{0} K\left(\operatorname{coh}_{C}^{\bar{G}}\left(Y_{N}\right)\right)$. Thus (8.10) is a free basis of $K\left(\operatorname{coh}_{C}^{\bar{G}}\left(Y_{N}\right)\right) / F_{0} K\left(\operatorname{coh}_{C}^{\bar{G}}\left(Y_{N}\right)\right)$ and therefore we have established (8.8).

Next we prove (8.9). Let $\mathrm{ZL}(V) \subset \mathrm{GL}(V)$ be the subgroup consisting of the non-zero scalar matrices and consider the multiplication map

$$
\mu: \mathrm{ZL}(V) \times \mathrm{SL}(V) \rightarrow \mathrm{GL}(V) .
$$

Then the kernel of $\mu$ is a group of order 2 generated by $(-I,-I)$. We put $\tilde{G}=\mu^{-1}(G)$ and let $H \subset \operatorname{SL}(V)$ be the image of $\tilde{G}$ with respect to the second projection. For any element $(z, h) \in \tilde{G}$, denote by $Z_{\tilde{G}}(z, h)$ and $Z_{G}(z h)$ the centralizers of $(z, h)$ in $\tilde{G}$ and $z h$ in $G$ respectively. Then the restriction $\mu: Z_{\tilde{G}}(z, h) \rightarrow Z_{G}(z h)$ is a surjective two-to-one map and hence the number of conjugates of $(z, h)$ coincides with the number of conjugates of $z h$. Therefore, the number of conjugacy classes in $\tilde{G}$ is twice the number of conjugacy classes in $G$. Thus we obtain

$$
\operatorname{rank} R(G)=\frac{1}{2} \operatorname{rank} R(\tilde{G}) .
$$

Moreover, since $\tilde{G} / Z \cong H$ and $Z$ is central in $\tilde{G}$, this can be written as

$$
\operatorname{rank} R(G)=\frac{1}{2} \operatorname{rank} R(H) \times|Z|=\operatorname{rank} R(H) \times|\bar{Z}| .
$$

Notice that $H$ acts on $V$ and $\bar{H}:=H / N \cong \bar{G} / \bar{Z} \subset \operatorname{PGL}(V)$ acts on $C=\mathbb{P}(V)$. Since $H$ is in $\operatorname{SL}(V)$, the McKay correspondence for the binary polyhedral (or dihedral) group $H$ establishes

$$
\sum_{k=1}^{3}\left|\bar{H}_{k}\right|=\operatorname{rank} R(H)+1
$$


where $\bar{H}_{k} \subset \bar{H}$ is the stabilizer of a point in $O_{k}$ (the left hand side of (8.14) is two plus the number of the irreducible exceptional curves in the minimal resolution of $V / H$, which is also the minimal resolution of $Y_{N} / \bar{H}$ ). Moreover, the isomorphism $\bar{H} \cong \bar{G} / \bar{Z}$ implies

$$
\left|\bar{H}_{k}\right| \times|\bar{Z}|=\left|\bar{G}_{k}\right|=\operatorname{rank} R\left(\bar{G}_{k}\right) \text {. }
$$

Putting the equalities (8.13), (8.14) and (8.15) together, we obtain (8.9). to

COROllary 1. The dual module $\operatorname{Hom}_{\mathbb{Z}}\left(F_{0} K\left(\operatorname{coh}_{C}^{\bar{G}}\left(Y_{N}\right)\right), \mathbb{Z}\right)$ is isomorphic

$$
\left\{\left(\theta_{1}, \theta_{2}, \theta_{3}\right) \in \bigoplus_{k=1}^{3} \operatorname{Hom}_{\mathbb{Z}}\left(R\left(\bar{G}_{k}\right), \mathbb{Z}\right)\left|\theta_{1}\right|_{\bar{Z}}=\left.\theta_{2}\right|_{\bar{Z}}=\left.\theta_{3}\right|_{\bar{Z}}\right\}
$$

\subsection{Main theorem.}

Proposition 5. Suppose a finite subgroup $G \subset \mathrm{GL}(2, \mathbb{C})$ contains $-I$ and $Y \rightarrow Y_{N} / \bar{G}$ is a resolution dominated by $Y_{\max }$. Then there exists a generic stability parameter $\theta \in \Theta$ such that $\mathscr{M}_{\theta} \cong Y$. Especially, the maximal resolution $Y_{\max }$ of $\left(\mathbb{C}^{2} / G, B\right)$ is isomorphic to the moduli space of $G$-constellations for some generic stability parameter $\theta$.

Proof. We may assume $G$ is non-abelian by Theorem 5 so we may apply the results of section 8.1. If we show there exists a generic parameter $\xi \in K\left(\operatorname{coh}^{\bar{G}}\left(Y_{N}\right)\right)_{\mathbb{Q}}^{0}$ such that $\mathscr{M}_{\xi}\left(Y_{N}\right) \cong Y$, then the assertion follows from Theorem 6 .

Let $P \in C$ be a point. Since $\bar{G}$ acts on $Y_{N} \times \mathbb{C}=\mathscr{M}_{\theta^{N}}(V \times \mathbb{C})$ and $\bar{Z}$ fixes $(P, 0), \bar{Z}$ acts on the Zariski tangent space $\tilde{T}:=T_{(P, 0)}\left(Y_{N} \times \mathbb{C}\right) \cong \mathbb{C}^{3}$ as a subgroup of $\operatorname{SL}(\tilde{T})$. Note that as a representation of $\bar{Z}, \tilde{T}$ is independent of the choice of the point $P$. Let $T^{\prime} \subset \tilde{T}$ be the two-dimensional $\bar{Z}$-invariant subspace transversal to $C$; then $\bar{Z} \subset \operatorname{SL}\left(T^{\prime}\right)$. Fix a generic stability parameter $\theta^{\bar{Z}} \in R(\bar{Z})_{\mathbb{Q}}^{*}$ for $\bar{Z}$-constellations (on $\tilde{T}$ ) satisfying $\theta^{\bar{Z}}(\mathbb{C}[\bar{Z}])=0$. Then $W:=$ $\mathscr{M}_{\theta^{\bar{Z}}}\left(T^{\prime}\right)$ is the minimal resolution of $T^{\prime} / \bar{Z}$. The Fourier-Mukai transform

$$
\varphi_{\theta^{\bar{Z}}}^{*}: R(\bar{Z})_{\mathbb{Q}}^{*} \cong K\left(\operatorname{coh}^{\bar{Z}}\left(T^{\prime}\right)\right)_{\mathbb{Q}} \stackrel{\sim}{\rightarrow} K(\operatorname{coh} W)_{\mathbb{Q}}
$$

sends $\theta^{\bar{z}}$ to an element $l_{\theta^{\bar{z}}}$ of $F^{1} K(\operatorname{coh} W)_{\mathbb{Q}} \cong \operatorname{Pic}(W)_{\mathbb{Q}}$ and it lies in the ample cone $\operatorname{Amp}(W)$ as in (2.1). (Notice that here $\operatorname{dim} T^{\prime}=2$ and $F^{2} K(\operatorname{coh} W)=$ 0.)

Take a point $P_{k}$ in the orbit $O_{k}$ for each $k \in\{1,2,3\}$. We consider the tangent spaces $\tilde{T}_{k}:=T_{\left(P_{k}, 0\right)}\left(Y_{N} \times \mathbb{C}\right)$ and $T_{k}=T_{P_{k}}\left(Y_{N}\right)$. Let $R_{k}$ denote the complete local ring of $T_{k} / \bar{G}_{k}$ at [0] which is isomorphic to the complete local 
ring of $Y_{N} / \bar{G}$ at $\left[P_{k}\right]$ :

$$
R_{k}:=\hat{\mathcal{O}}_{T_{k} / \bar{G}_{k},[0]} \cong \hat{\mathcal{O}}_{Y_{N} / \bar{G},\left[P_{k}\right]} .
$$

By this isomorphism, there is a resolution

$$
Y_{k} \rightarrow T_{k} / \bar{G}_{k}
$$

with an isomorphism

$$
Y_{k} \times_{\left(T_{k} / \bar{G}_{k}\right)} \operatorname{Spec} R_{k} \cong Y \times_{\left(Y_{N} / \bar{G}\right)} \operatorname{Spec} R_{k}
$$

over Spec $R_{k}$. Since $\bar{G}_{k}$ is abelian, we can apply Proposition 2 where the first factor of $T_{k} \cong \mathbb{C}^{2}$ is $T_{P_{k}}(C)$ (so that $(1,0)$ lies in $T_{P_{k}}(C)$ and $G_{(1,0)}=\bar{Z}$ ) and obtain a projective crepant resolution

$$
U_{\Sigma_{k}} \rightarrow \tilde{T}_{k} / \bar{G}_{k}
$$

such that $Y_{k} \subset U_{\Sigma_{k}}$ and that the restriction map $\operatorname{Amp}\left(U_{\Sigma_{k}}\right) \rightarrow \operatorname{Amp}(W)$ is surjective. Choose a class $l_{k} \in \operatorname{Amp}\left(U_{\Sigma_{k}}\right)$ which is mapped to $l_{\theta_{\bar{z}}} \in \operatorname{Amp}(W)$ for each $k$. Then by Theorem 2 we can find a generic stability parameter $\theta_{k}$ for $\bar{G}_{k}$-constellations on $\tilde{T}_{k}$ such that $\mathscr{M}_{\theta_{k}}\left(\tilde{T}_{k}\right) \cong U_{\Sigma_{k}}$ and the class of $\varphi_{\theta_{k}}^{*}\left(\theta_{k}\right)$ in $\operatorname{Pic}\left(U_{\Sigma_{k}}\right)_{\mathbb{Q}}$ coincides with $l_{k}$. Since $\left[\varphi_{\theta_{k}}^{*}\left(\theta_{k}\right)\right]=l_{k}$ and $l_{k}$ restricts to $l_{\theta^{\bar{z}}}, \theta_{k}$ restricts to $\theta^{\bar{Z}}$ on $R(\bar{Z})$. Then Corollary 1 shows that $\left(\theta_{1}, \theta_{2}, \theta_{3}\right)$ determines an element of $F_{0} K\left(\operatorname{coh}_{C}^{\bar{G}}\left(Y_{N}\right)\right)_{\mathbb{Q}}^{*}$. Lift it to an element $\xi \in K\left(\operatorname{coh}^{\bar{G}}\left(Y_{N}\right)\right)_{\mathbb{Q}} \cong$ $K\left(\operatorname{coh}_{C}^{\bar{G}}\left(Y_{N}\right)\right)_{\mathbb{Q}}^{*}$. Since the restriction of $\xi$ to $K\left(\operatorname{coh}^{\bar{G}}\left(O_{k}\right)\right)_{\mathbb{Q}} \cong R\left(\bar{G}_{k}\right)_{\mathbb{Q}}^{*}$ is $\theta_{k}$ which is of rank 0 , we have rank $\xi=0$ and we can consider the moduli space $\mathscr{M}_{\xi}\left(Y_{N}\right)$.

We claim that there is an isomorphism

$$
\mathscr{M}_{\xi}\left(Y_{N}\right) \times_{\left(Y_{N} / \bar{G}\right)} \operatorname{Spec} R_{k} \cong \mathscr{M}_{\theta_{k}}\left(T_{k}\right) \times_{\left(T_{k} / \bar{G}_{k}\right)} \operatorname{Spec} R_{k}
$$

over $\operatorname{Spec} R_{k}$. For any locally noetherian scheme $S$ over $\operatorname{Spec} R_{k}$, an $S$-valued point of the left hand side of (8.17) is given by a flat family of $\xi$-stable $\bar{G}$-constellations on $Y_{k}$ parameterized by $S$, which is an object of $\operatorname{coh}^{\bar{G}}\left(Y_{N} \times_{\left(Y_{N} / \bar{G}\right)} S\right)$. Similarly, an $S$-valued point of the right hand side of (8.17) is given by a flat family of $\theta_{k}$-stable $\bar{G}_{k}$-constellations on $T_{k}$ parameterized by $S$, which is an object of $\operatorname{coh}^{\bar{G}_{k}}\left(T_{k} \times{ }_{\left(T_{k} / \bar{G}_{k}\right)} S\right)$.

Notice that

$$
\begin{aligned}
Y_{N} \times_{\left(Y_{N} / \bar{G}\right)} S & \cong\left(Y_{N} \times_{\left(Y_{N} / \bar{G}\right)} \operatorname{Spec} R_{k}\right) \times_{\left(\operatorname{Spec} R_{k}\right)} S \\
& \cong\left(\coprod_{Q \in O_{k}} \operatorname{Spec} \hat{\mathcal{O}}_{Y_{N}, Q}\right) \times_{\left(\operatorname{Spec} R_{k}\right)} S \\
& \supset \operatorname{Spec} \hat{\mathcal{O}}_{Y_{N}, P_{k}} \times_{\left(\operatorname{Spec} R_{k}\right)} S
\end{aligned}
$$




$$
\begin{aligned}
& \cong \operatorname{Spec} \hat{\mathcal{O}}_{T_{k}, 0} \times_{\left(\operatorname{Spec} R_{k}\right)} S \\
& \cong T_{k} \times_{\left(T_{k} / \bar{G}_{k}\right)} S
\end{aligned}
$$

which induces an equivalence

$$
\operatorname{coh}^{\bar{G}}\left(Y_{N} \times_{\left(Y_{N} / \bar{G}\right)} S\right) \cong \operatorname{coh}^{\bar{G}_{k}}\left(T_{k} \times_{\left(T_{k} / \bar{G}_{k}\right)} S\right)
$$

(this is almost the same as (8.3)). This equivalence gives a bijection between $S$-valued points of the both sides of (8.17) and we obtain (8.17).

Our choice of $\theta_{k}$ implies $\mathscr{M}_{\theta_{k}}\left(T_{k}\right) \cong Y_{k}$ and hence (8.16) and (8.17) yield an isomorphism

$$
\mathscr{M}_{\xi}\left(Y_{N}\right) \times_{\left(Y_{N} / \bar{G}\right)} \operatorname{Spec} R_{k} \cong Y \times_{\left(Y_{N} / \bar{G}\right)} \operatorname{Spec} R_{k} .
$$

over Spec $R_{k}$. Since $\mathscr{M}_{\xi}\left(Y_{N}\right)$ and $Y$ are both isomorphic to $Y_{N} / \bar{G}$ except over the points $\left[P_{1}\right],\left[P_{2}\right]$, and $\left[P_{3}\right]$, we obtain $\mathscr{M}_{\xi}\left(Y_{N}\right) \cong Y$.

Recall that we say $G \subset \operatorname{GL}(2, \mathbb{C})$ is small if $G$ acts freely on $\mathbb{C}^{2} \backslash\{0\}$. The following lemma follows from the classification of small subgroups of $\operatorname{GL}(2, \mathbb{C})$ but we give a proof for the reader's sake.

Lemma 5. If a finite small subgroup $G \subset \mathrm{GL}(2, \mathbb{C})$ is non-abelian, then it contains $-I$ as a unique element of order 2.

Proof. If $G$ is non-abelian, then its image $G^{\prime} \subset \operatorname{PGL}(2, \mathbb{C})$ is also nonabelian and therefore it is either a dihedral or a polyhedral group. Especially, the orders $\left|G^{\prime}\right|$ and $|G|$ are even. Then $G$ contains an element of order 2. If it is not $-I$, then it fixes a line in $\mathbb{C}^{2}$, contradicting the smallness of $G$.

THEOREM 7. If $G \subset \mathrm{GL}(2, \mathbb{C})$ is a finite small subgroup, then Conjecture 4 is true.

Proof. The abelian case follows from Theorem 5. Otherwise, $G$ contains $-I$ by the above lemma. Moreover, the minimal resolution of $V / G$ factors through $Y_{N} / \bar{G}$; see [Bri68]. Then the assertion follows from Proposition 5 .

\section{References}

[BCZ17] Arend Bayer, Alastair Craw and Ziyu Zhang, Nef divisors for moduli spaces of complexes with compact support, Selecta Math. (N.S.) 23 (2017), no. 2, 1507-1561. MR 3624918

[BKR01] Tom Bridgeland, Alastair King and Miles Reid, The McKay correspondence as an equivalence of derived categories, J. Amer. Math. Soc. 14 (2001), no. 3, 535-554 (electronic). MR MR1824990 (2002f:14023) 
[Bri68] Egbert Brieskorn, Rationale Singularitäten komplexer Flächen, Invent. Math. 4 (1967/1968), 336-358. MR 0222084 (36 \#5136)

[Bri99] Tom Bridgeland, Equivalences of triangulated categories and Fourier-Mukai transforms, Bull. London Math. Soc. 31 (1999), no. 1, 25-34. MR MR1651025 (99k:18014)

[Bri07] Tom Bridgeland, Stability conditions on triangulated categories, Ann. of Math. (2) 166 (2007), no. 2, 317-345. MR 2373143 (2009c:14026)

[CI04] Alastair Craw and Akira Ishii, Flops of $G$-Hilb and equivalences of derived categories by variation of GIT quotient, Duke Math. J. 124 (2004), no. 2, 259307. MR MR2078369

[IINdC13] Akira Ishii, Yukari Ito and Álvaro Nolla de Celis, On $G / N$-Hilb of $N$-Hilb, Kyoto J. Math. 53 (2013), no. 1, 91-130. MR 3049308

[Ish02] Akira Ishii, On the McKay correspondence for a finite small subgroup of GL(2, $\mathbb{C})$, J. Reine Angew. Math. 549 (2002), 221-233. MR MR1916656 (2003d:14021)

[IU15] Akira Ishii and Kazushi Ueda, The special McKay correspondence and exceptional collections, Tohoku Math. J. (2) 67 (2015), no. 4, 585-609. MR 3436544

[Jun16] Seung-Jo Jung, Terminal quotient singularities in dimension three via variation of GIT, J. Algebra 468 (2016), 354-394. MR 3550869

[Jun18] Seung-Jo Jung, On the Craw-Ishii conjecture, J. Pure Appl. Algebra 222 (2018), no. 7, 1579-1605. MR 3763272

[Kaw18] Yujiro Kawamata, Derived McKay correspondence for GL(3,C), Adv. Math. 328 (2018), 1199-1216. MR 3771150

[Kin94] Alastair King, Moduli of representations of finite-dimensional algebras, Quart. J. Math. Oxford Ser. (2) 45 (1994), no. 180, 515-530. MR MR1315461 (96a:16009)

[Kę14] Oskar Kędzierski, Danilov's resolution and representations of the McKay quiver, Tohoku Math. J. (2) 66 (2014), no. 3, 355-375. MR 3266737

[KM98] János Kollár and Shigefumi Mori, Birational geometry of algebraic varieties, Cambridge Tracts in Mathematics, vol. 134, Cambridge University Press, Cambridge, 1998, With the collaboration of C. H. Clemens and A. Corti, Translated from the 1998 Japanese original. MR 1658959

[KSB88] János Kollár and Nicholas I. Shepherd-Barron, Threefolds and deformations of surface singularities, Invent. Math. 91 (1988), no. 2, 299-338. MR 922803

[NdCS17] Álvaro Nolla de Celis and Yuhi Sekiya, Flops and mutations for crepant resolutions of polyhedral singularities, Asian J. Math. 21 (2017), no. 1, 1-45. MR 3632435

\author{
Akira Ishii \\ Graduate School of Mathematics \\ Nagoya University \\ Furocho, Chikusaku, Nagoya, 464-8602 Japan \\ E-mail: akira141@math.nagoya-u.ac.jp
}

